\section{Cervical epidural hematoma with Brown-Sequard syndrome caused by an epidural injection: a case report}

\author{
Young Jun Cho, Haewon Jung, Sungbae Moon, Hyun Wook Ryoo \\ Department of Emergency Medicine, Kyungpook National University School of Medicine, Daegu, Korea
}

Epidural hematoma with Brown-Sequard syndrome caused by an epidural injection is a rarely found condition in the emergency department (ED). We report an unusual case of Brown-Sequard syndrome in a 55-year-old man who presented at the ED with right-sided weakness and contralateral loss of pain and temperature sensation after a cervical epidural injection for shoulder pain. Cervicla spine magnetic resonance imaging showed an epidural hematoma from $\mathrm{C} 4$ to C6. After admission, his right hemiparesis and contralateral sensory loss improved within eight days, and surgical decompression was not required. Diagnosing spinal lesions in the ED is challenging, especially in patients with acute neurological signs requiring immediate evaluation for stroke. In this case, definite hemiparesis and some contralateral sensory loss were noted. Therefore, a potential spinal lesion was suspected rather than a stroke. This case emphasized the importance of conducting a focused neurological examination after history taking.

Keywords Hematoma; Brown-Sequard syndrome; Epidural injections; Case reports

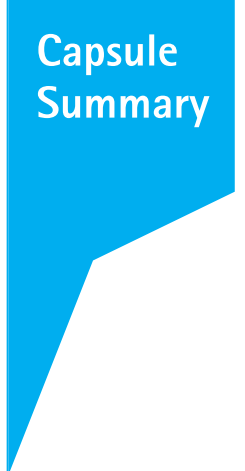

eISSN: 2383-4625

Received: 13 August 2020

Revised: 29 October 2020

Accepted: 25 November 2020

Correspondence to: Haewon Jung Department of Emergency Medicine, Kyungpook National University School of Medicine, 130 Dongdeok-ro, Junggu, Daegu 41944, Korea

E-mail: blueseahw@naver.com ORCID

https://orcid.org/0000-0002-2303-3142

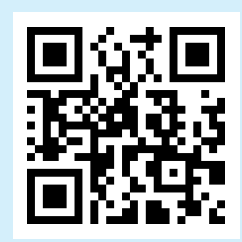

How to cite this article:

Cho YJ, Jung H, Moon S, Ryoo HW. Cervical epidural hematoma with Brown-Sequard syndrome caused by an epidural injection: a case report. Clin Exp Emerg Med 2021;8(4):336-339. https://doi. org/10.15441/ceem.20.104

This is an Open Access article distributed under the terms of the Creative Commons Attribution Non-Commercial License (https:// creativecommons.org/licenses/by-nc/4.0/). 


\section{INTRODUCTION}

Chronic pain due to cervical radiculopathy is a common condition. Drug treatment is primarily used for treating this condition, and epidural injections are known to be effective in relieving the pain. ${ }^{1}$ However, epidural injections may cause various unexpected complications such as epidural hematomas (EHs). ${ }^{2}$ If an EH leads to severe neurologic symptoms or progresses rapidly, surgical decompression may be required. ${ }^{3}$ Additionally, Brown-Sequard syndrome (BSS) may occur due to an EH. However, BSS is generally known to occur due to a penetrating spinal cord injury, and the number of non-traumatic BSS cases is significantly less. ${ }^{5}$ In this case report, we describe a rare EH case with BSS, presented to the emergency department (ED).

\section{CASE REPORT}

A 55-year-old man presented to the ED with a gait disturbance and right-sided weakness. His risk factors for acute stroke included hypertension, smoking, and cardiovascular problems. He was taking aspirin and clopidogrel for a cardiovascular disease. On arrival at the ED, his vital signs were as follows: blood pressure, 165/95 $\mathrm{mmHg}$; respiratory rate, 16 breaths/min; heart rate, 78/ min; body temperature, $36.7^{\circ} \mathrm{C}$; and oxygen saturation with room air, 98\%. His mental status was alert without any disorientation or speech disturbance, and his Glasgow Coma Scale was 15. Neurological examination revealed normal cranial nerve functions, but motor weakness (Grade 4) was confirmed in the right arm

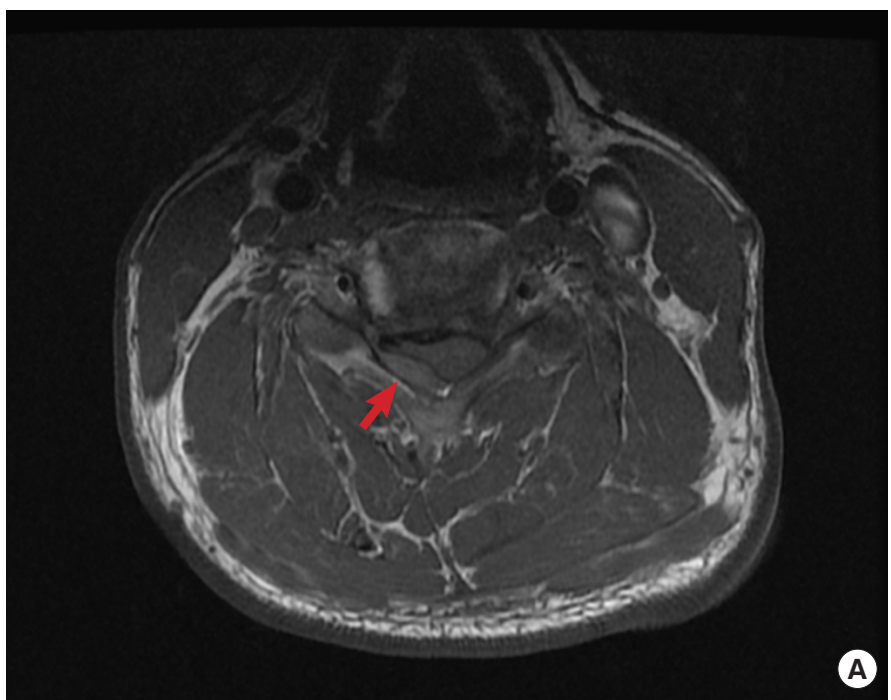

and leg. Symptoms developed within 3 hours, and we consulted the in-hospital stroke team.

Brain computed tomography, angiography, and magnetic resonance imaging were performed sequentially to rule out vascular and brain lesions, but no specific findings were observed. After

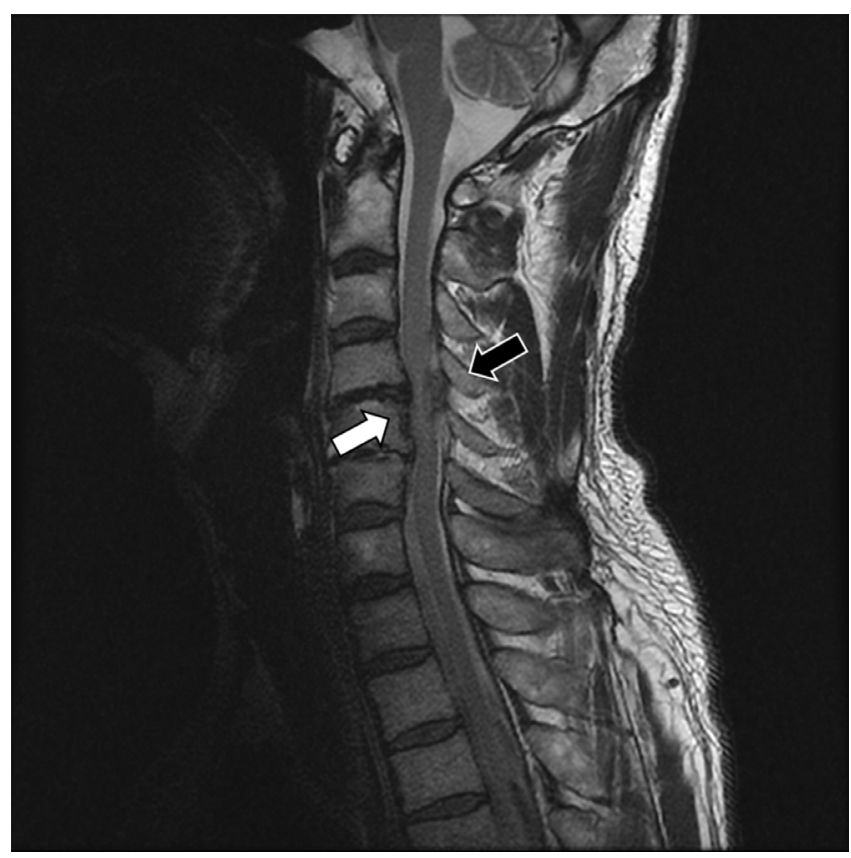

Fig. 1. T2 weighted sagittal view of the cervical spine magnetic resonance imaging showing the ossification of the posterior longitudinal ligament (white arrow) and acute epidural hematoma (black arrow). Informed consent for publication of the clinical images was obtained from the patient.

Fig. 2. T1 weighted axial view (A), T2 weighted axial view (B) of the cervical spine magnetic resonance imaging demonstrating compressive myelopathy of the right hemicord caused by an acute epidural hematoma (red arrow). Informed consent for publication of the clinical images was obtained from the patient. 


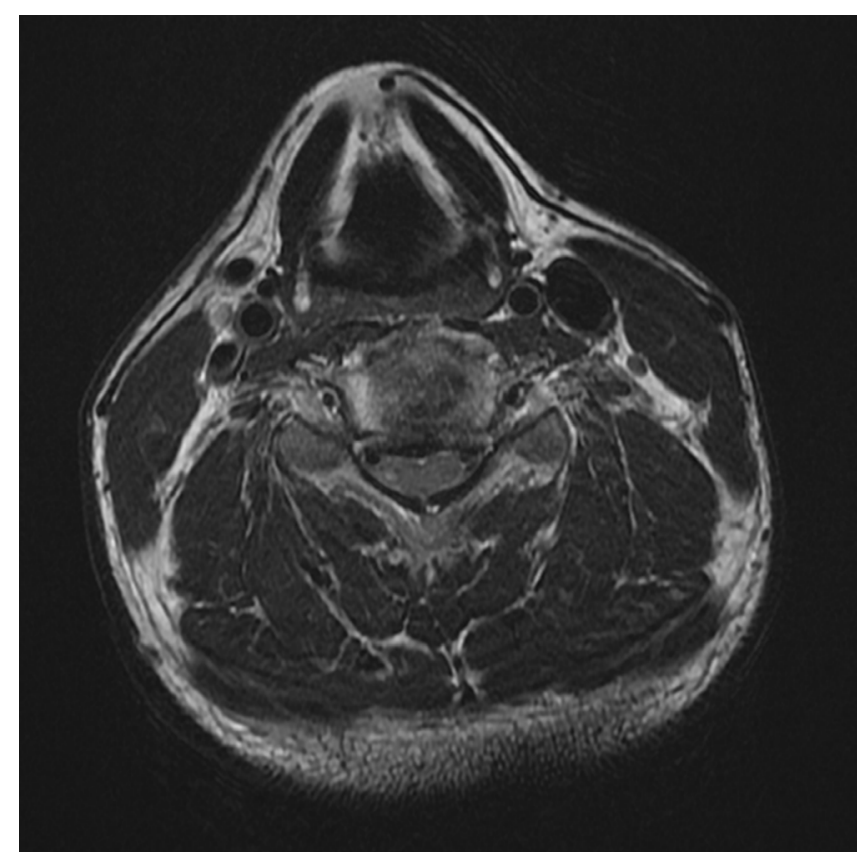

Fig. 3. T2 weighted axial view of the cervical spine magnetic resonance image, 2 months after follow-up study, shows complete epidural hematoma resorption. Informed consent for publication of the clinical images was obtained from the patient.

the neuroimaging, at the time of preparing thrombolytic therapy, a full neurological examination was performed. Left hemibody pain and temperature sensation below T5 was slightly impaired (90\% of normal sensation). There was no hyperreflexia or clonus, and the anal sphincter tone was normal. The patient was given a C4-C5 transforaminal cervical epidural injection 30 minutes before the onset of neurological symptoms at a local pain clinic by a physician specialized in rehabilitation medicine with radiological assistance (C-arm fluoroscopy). Considering the possibility of spinal cord complications due to the epidural injection by neurological examination, no thrombolytic therapy was given. Subsequently, cervical spine magnetic resonance imaging was performed. Mild ossification of the posterior longitudinal ligament was seen at the C4-5-6 level (Fig. 1), and a hematoma at the C45-6 level was also confirmed (Fig. 2). Because the right-sided weakness was not severe at the time of admission, surgical intervention was not pursued as a result of ED consultation from the neurosurgeon. The patient was admitted to the general ward and closely monitored. The patient gradually improved with conservative care. Gait was regained four days after admission, and weakness improved six days later. The sensory loss was almost completely recovered (95\% of normal sensation) when he was discharged eight days after admission. The 2-month follow-up imaging study showed complete resorption of EH (Fig. 3).

\section{DISCUSSION}

Various complications may develop following a cervical epidural injection. ${ }^{2}$ In general, the majority are self-limited minor complications such as a vasovagal reaction or procedure-related pain. However, an improper needle position can cause serious problems such as a direct spinal cord injury by the needle, epidural abscess, and EH. Embolic infarction and brain stem herniation due to an intra-arterial injection may also occur. ${ }^{6}$ Spinal EH is treated by conservative treatment if the initial condition is good. However, if neurological symptoms progress rapidly or severe paralysis such as para or quadriparesis is present, urgent surgical decompression may be necessary. ${ }^{3}$ Therefore, in patients with progressive complications caused by cervical lesions, treatment must be performed urgently with caution.

BSS is usually caused by penetrating spinal cord injuries such as gunshot and stab wounds. Symptoms are characterized by ipsilateral weakness and contralateral loss of pain and temperature two levels below the lesion due to lateral spinothalamic tract damage. Tumor, disc herniation, infection, autoimmune disease, and non-penetrating trauma resulting in an $\mathrm{EH}$ may also cause BSS. EH incidence after epidural injections is 1 in 150,000, but EH with BSS is more unusual. ${ }^{6,7}$

There are many difficulties in ensuring early diagnosis of cervical spine lesions in patients visiting the ED. It takes a substantial amount of time to perform a full neurologic examination in a patient visiting the ED. This can be very restrictive when imaging should be performed immediately to distinguish brain lesions. Because stroke treatment is time sensitive, the ED should reduce inhospital time delays, such as those associated with door-to-imaging and door-to-needle times through a critical pathway. Due to the required tests and procedures, there is limited time to perform a full neurological examination on a patient. Nevertheless, we learned with this case that emergency physicians should be cautious to exam a patient who complains neurological symptoms.

The epidural injection is useful for treating spinal radiculopathy. ${ }^{8}$ This case illustrates that BSS caused by an EH is possible after an epidural injection. In patients presenting with stroke symptoms and epidural injection history, it may be desirable to carry out a focused neurological examination at the beginning with the possibility of spinal cord lesions.

\section{CONFLICT OF INTEREST}

No potential conflict of interest relevant to this article was reported. 


\section{REFERENCES}

1. Woods BI, Hilibrand AS. Cervical radiculopathy: epidemiology, etiology, diagnosis, and treatment. J Spinal Disord Tech 2015; 28:E251-9.

2. El-Yahchouchi CA, Plastaras CT, Maus TP, et al. Adverse event rates associated with transforaminal and interlaminar epidural steroid injections: a multi-institutional study. Pain Med 2016; 17:239-49.

3. Domenicucci M, Mancarella C, Santoro G, et al. Spinal epidural hematomas: personal experience and literature review of more than 1000 cases. J Neurosurg Spine 2017;27:198208.

4. Ofluoglu E, Ozdemir A, Toplamaoglu H, Sofuoglu E. Spontaneous cervical epidural hematoma causing Brown-Sequard syn- drome: case report. Turk Neurosurg 2009;19:99-102.

5. Borges LF, Zervas NT, Lehrich JR. Idiopathic spinal cord herniation: a treatable cause of the Brown-Sequard syndrome: case report. Neurosurgery 1995;36:1028-33.

6. Schneider BJ, Maybin S, Sturos E. Safety and complications of cervical epidural steroid injections. Phys Med Rehabil Clin N Am 2018;29:155-69.

7. Kashyap S, Majeed G, Lawandy S. A rare case of Brown-Sequard syndrome caused by traumatic cervical epidural hematoma. Surg Neurol Int 2018;9:213.

8. Manchikanti L, Nampiaparampil DE, Candido KD, et al. Do cervical epidural injections provide long-term relief in neck and upper extremity pain: a systematic review. Pain Physician 2015; 18:39-60. 